\title{
TRANSIENT PHENOMENA IN SPUTTERED CdS PHOTOCELLS
}

\author{
A. PIEL and H. MURRAY \\ Laboratoire d'Electronique et d'Automatique U.E.R. des Sciences et Techniques B.P. 400676077 \\ Le Havre Cedex France
}

(Received June 30, 1979; in final form April 23, 1980)

\begin{abstract}
We describe experimental data and theoretical studies of response time measurements in sandwich structures $\mathrm{Al}-\mathrm{CdS}-\mathrm{Au}$. The speed of response is about $2 \mu \mathrm{s}$ in a large sensitivity range. A theoretical simplified model shows that this response time is limited by the majority carrier lifetime. The concept of photoconductive gain $G$ which represents the ratio between the carrier lifetime $\tau_{n}$ and the transit time $t_{n}$ seems to be particularly well adapted to describe specific properties of very thin photovoltaic cells.
\end{abstract}

\section{INTRODUCTION}

If semi-conductor devices are particularly required in optoelectronics, they present in some cases, a high response time or memory effects, which are unsuitable for high frequency modulated light reception. This low speed of response is attributed to trapping phenomena in doped materials and generally, there is some difficulty to obtain high-rate photocurrent and fast response time together. ${ }^{1}$

Photovoltaic cells can be prepared from sputtered metal-photoconductor-metal sandwich structures. ${ }^{2}$ These photovoltaïc effects have been attributed to interband transitions and photoionisation of trapped centres. $^{2}$ The most important effect, for the energy efficiency, is due to the transport of photoexcited carriers by the built-in electric field arising from the difference between metallic work functions.

A simplified model ${ }^{3}$ explains this effect using the increase in conductivity $\sigma$ caused by the photoexcited free carriers which gives $\sigma_{p h}=e\left(\mu_{n} n+\mu_{p} p\right)=$ $e g\left(\mu_{n} \tau_{n}+\mu_{p} \tau_{p}\right)$. This present work describe lifetimes measurement from the response time with pulse light irradiation. A simple theoretical study, from continuity equations ${ }^{4}$ shows that the response time is limited by the majority carrier lifetime which has a value which has been estimated less than $5 \mu \mathrm{s}$. This study gives complementary knowledge on the photoeffects in very thin sandwich structures.

\section{EXPERIMENTS AND RESULTS}

Sandwich structures are deposited by Radio Frequency sputtering from high purity target, as shown on Table I.

The substrate consists of a glass patch optically polished. The photoconductor is sputtered so as to obtain a very thin film $(600 \AA)$ with a high built-in electric field which arises from the different work functions of aluminium and gold electrodes. The capacitance of this photocell is $20 \mathrm{nF}$.

After thermal and electrical stabilization, the photovoltaïc effect, arising from electron photon

TABLE I

Deposition processes

\begin{tabular}{llll}
\hline $\begin{array}{l}\text { Deposition } \\
\text { process }\end{array}$ & $\begin{array}{l}\text { Aluminium } \\
\text { R.F.sputtering }\end{array}$ & $\begin{array}{l}\text { Cadmium sulphide } \\
\text { R.F. sputtering }\end{array}$ & $\begin{array}{l}\text { Gold thermal } \\
\text { evaporation }\end{array}$ \\
\hline thickness & $1200 \AA$ & $600 \AA$ & $150 \AA$ \\
\hline $\begin{array}{l}\text { Deposition } \\
\text { source purity }\end{array}$ & target & target & wire \\
\hline
\end{tabular}


interaction induces a short-circuit current ${ }^{2}$ which presents linear variations with the intensity of light from $10^{-6} \mathrm{~W} / \mathrm{cm}^{2}$ to $0.1 \mathrm{~W} / \mathrm{cm}^{2}$.

The spectral sensitivity has been previously studied and explained from the simplified quantum theory of photon electron interaction. ${ }^{3,4}$

The response-time measurement has been made from the light pulse of a mercury stroboscopic lamp. The short-circuit current is measured from the voltage induced in a variable load resistance. These experimental processes are reported on Figure 1. The photoresponse has been studied with different values of the load resistance, and with the lowest value $R=10 \Omega$, the response-time is about $2 \mu \mathrm{s}$. Figures 2 and 3 show the oscillograms and the variations of time response versus the load resistance $R$.

\section{RESPONSE-TIME MEASUREMENTS}

When the illumination is a time dependent function, the current densities are given by the continuity equations ${ }^{4}$

$$
\begin{aligned}
& \frac{\partial n}{\partial t}=\frac{1}{e} \frac{\partial J_{n}}{\partial x}+g(x, t)-\frac{n}{\tau_{n}} \\
& \frac{\partial p}{\partial t}=-\frac{1}{e} \frac{\partial J_{p}}{\partial x}+g(x, t)-\frac{p}{\tau_{p}}
\end{aligned}
$$
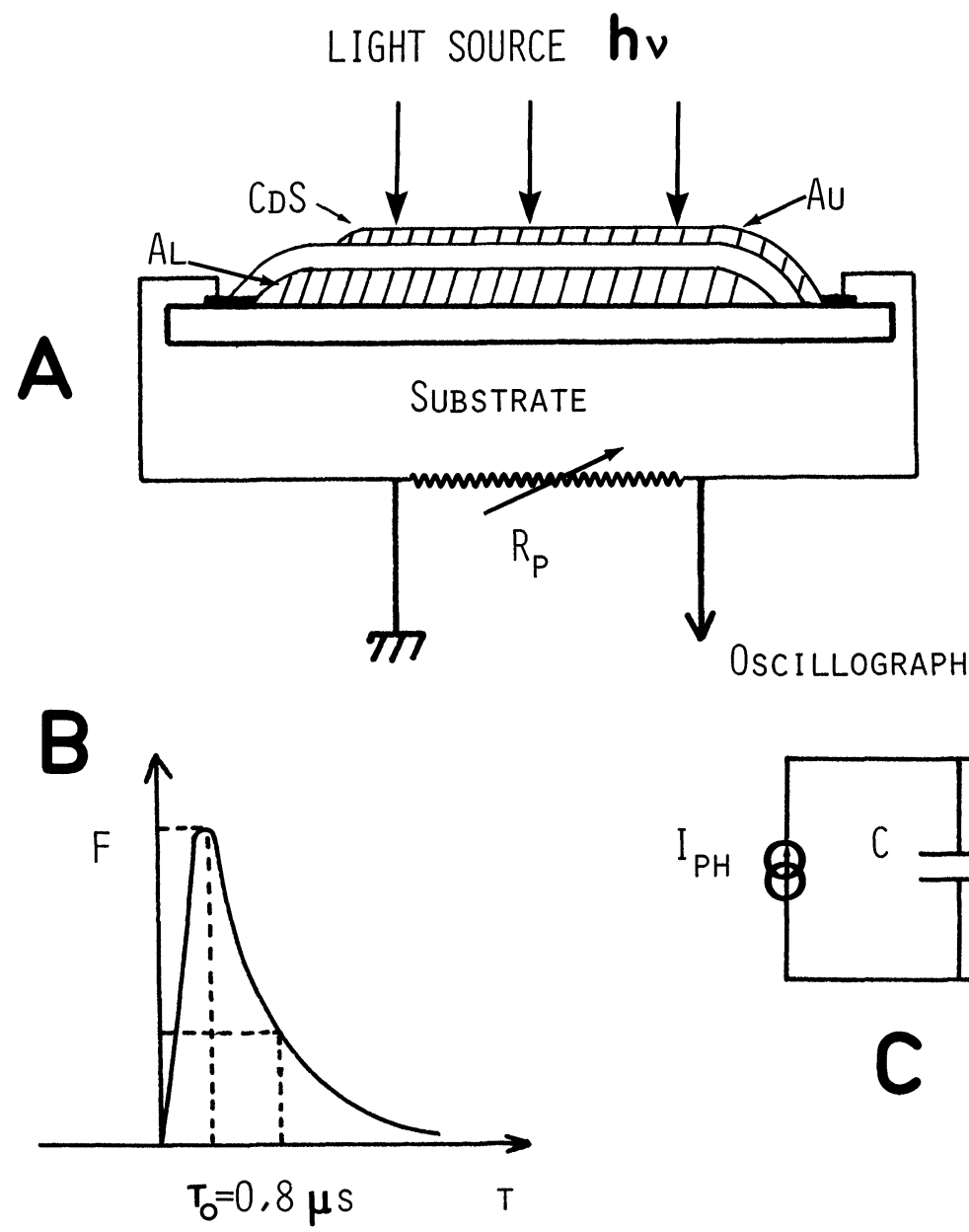

FIGURE 1 Experimental processes, A) Photoelectric device, B) Light pulse of mercury stroboscopic lamp, C) Equivalent circuit of $\mathrm{Al} / \mathrm{CdS} / \mathrm{Au}$ photocell. 


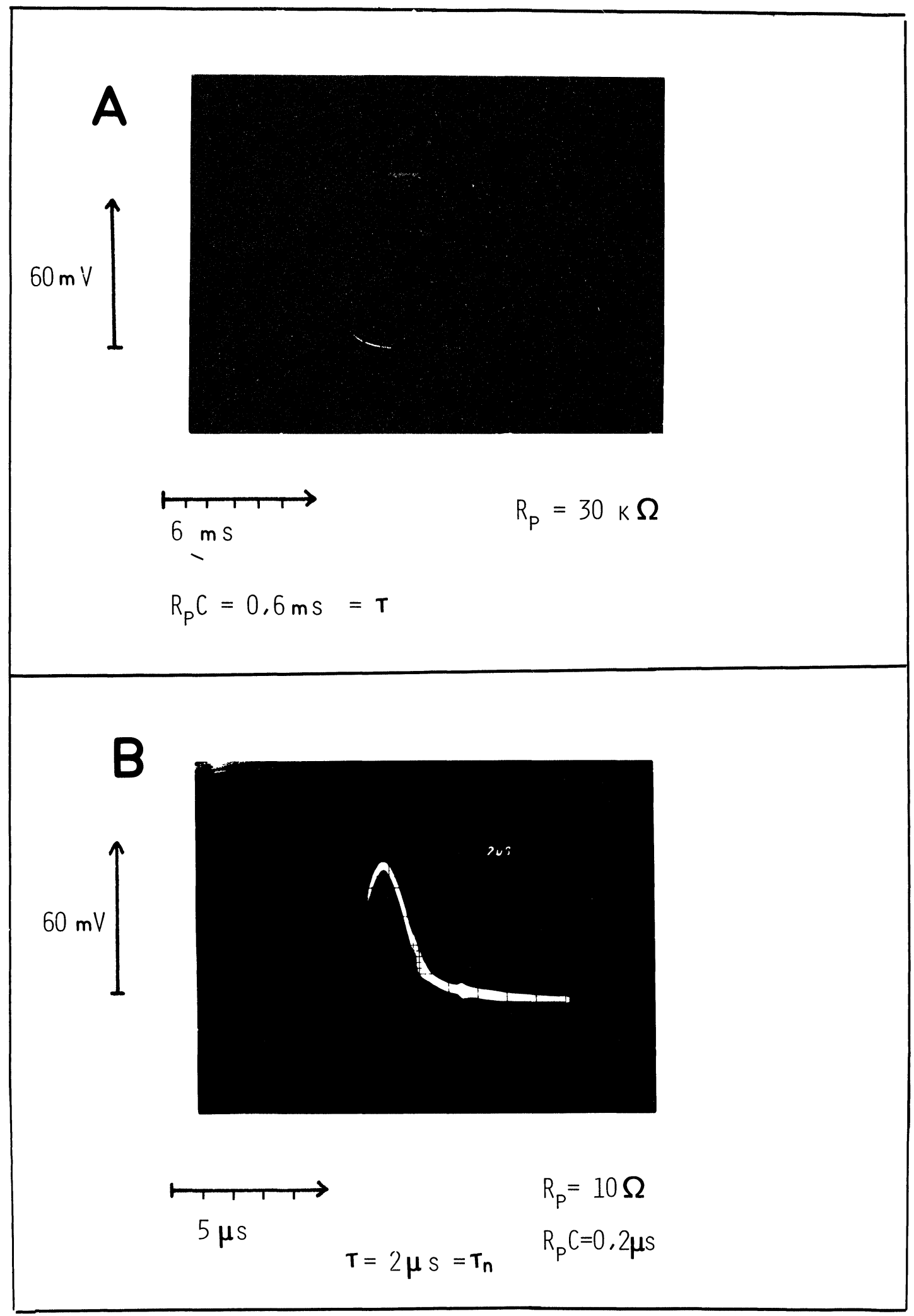

FIGURE 2 Photoresponse of sandwich photocells $V=R_{p} I_{p h}=R_{p} I_{0} \exp t / \tau$ A) $R_{p}=30 \mathrm{k} \Omega \tau=R_{p} C$ B) $R_{p}=$ $10 \Omega \tau=\tau_{n}=2 \mu \mathrm{s}$. 
where

$n$ and $p$ are electrons and holes concentrations

$J_{n}$ and $J_{p}$ are electrons and holes current densities

$\tau_{n}$ and $\tau_{p}$ are electrons and holes lifetimes

$g(x, t)$ is number of electrons-holes pairs created by illumination.

The current densities $J_{n}$ and $J_{p}$ can be obtained from the expression of drift and diffusion current. The differential system has been solved by Laplace transform (given in the appendix) with the following hypothesis:

$-g(x, t)=g(t)$ (i.e. is independent of $x$ for very low thickness $(L<0.1 \mu \mathrm{m}))$

- The electric field is independent of $x$ (no capacitance variations)

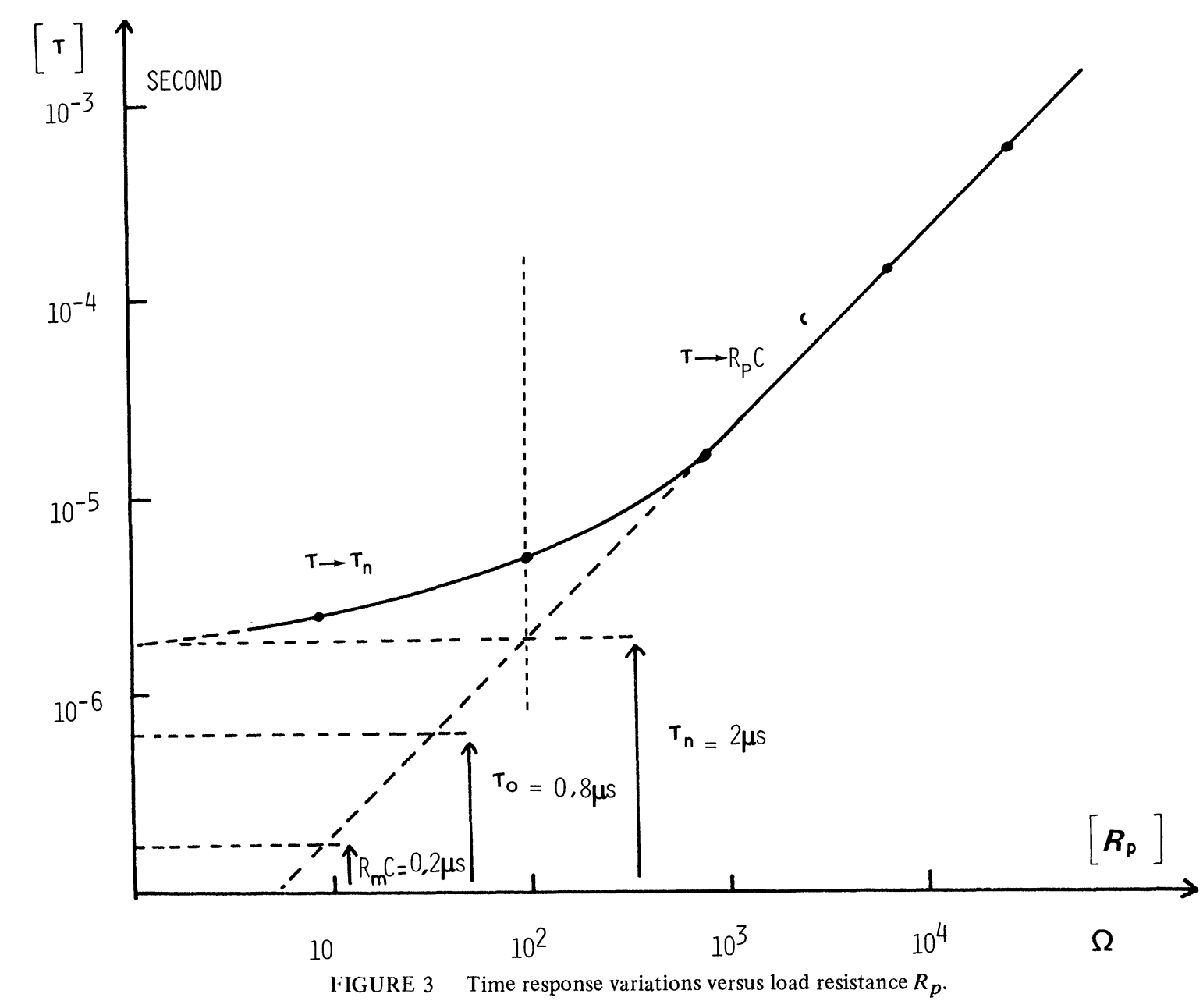

FIGURE 3 Time response variations versus load resistance $R_{p}$.
The photocurrent density is then given by:

$$
\begin{aligned}
& \text { for } a \delta(t) \text { function }\left\{\begin{array}{l}
g(t)=\frac{g_{0}}{\tau_{0}} \quad 0<t<\tau_{0} \\
g(t)=0 \quad \tau_{0}<t
\end{array}\right. \\
& J_{p h}=e E g_{0}\left(\mu_{n} \exp ^{-t / \tau_{n}}+\mu_{p} \exp ^{-t / \tau_{p}}\right)
\end{aligned}
$$

for an exponential decay $g(t)=g_{0} \exp ^{-t / \tau_{0}}$

$$
\begin{aligned}
J_{p h}(t)=e E g_{0} & {\left[\mu_{n} \frac{\tau_{n} \exp ^{-t / \tau_{n}-\tau_{0} \exp ^{-t / \tau_{0}}}}{\tau_{n}-\tau_{0}}\right.} \\
& \left.+\mu_{p} \frac{\tau_{p} \exp ^{-t / \tau_{p}-\tau_{0} \exp ^{-t / \tau_{0}}}}{\tau_{p}-\tau_{0}}\right]
\end{aligned}
$$

As cadmium sulphide is a $n$ type sem:-conductor, we 
can write, with a good approximation:

$$
J_{p h}=e E g_{0} \quad \mu_{n} \frac{\tau_{n} \exp ^{-t / \tau_{n}}-\tau_{0} \exp ^{-t / \tau_{0}}}{\tau_{n}-\tau_{0}}
$$

The total current in the load resistance (Figure 1) is given by the differential equation

$$
R C \frac{d I(t)}{d t}+I(t)=I_{p h}=J_{p h} \quad \frac{1}{S}
$$

with the solution:

$$
\begin{aligned}
I(t)=I_{0} \frac{(R C)^{2} \exp ^{-t / R C}}{\left(\tau_{0}-R C\right)\left(\tau_{n}-R C\right)} & +\frac{\tau_{0}{ }^{2} \exp ^{-t / \tau_{0}}}{\left(\tau_{0}-\tau_{n}\right)\left(\tau_{0}-R C\right)} \\
& +\frac{\tau_{n}{ }^{2} \exp ^{-t / \tau_{n}}}{\left(\tau_{n}-\tau_{0}\right)\left(\tau_{n}-R C\right)}
\end{aligned}
$$

This expression can be approximated by $I(t)=I_{0} \exp ^{-t / R C}$ for $R C>\tau_{n}$ and

$$
I(t)=I_{0} \exp ^{-t / \tau_{n}} \text { for } \tau_{n}>\tau_{0}>R C
$$

The extrapolation of response-time variations versus load resistance $R$ to $R<10 \Omega$ gives the rough value of $\tau=2 \mu$ s for $\tau_{n}$. As the light pulse has a decay time less than $0.8 \mu \mathrm{s}$, the photoresponse time of cadmium sulphide can be estimated to be within an order of magnitude of $2 \mu \mathrm{s}$.

Furthermore, even if it is possible to implement a better accuracy with other experimental processes, ${ }^{5}$ for example modulated light by Kerr cell, the photoresponse time measurement would be limited by the internal $R C$ time constant. The semitransparent metallic gold electrode requires a maximum thickness of $150 \AA$, and in this condition, the total metallic resistance which cannot be reduced below $5 \Omega$ sets the limit of the accuracy from the time constant $R C=0.1 \mu \mathrm{s}$

To sum up we attribute a value of approx. $2 \mu$ s to $\tau_{n}$, and even if the accuracy of this measurement is about $20 \%$, it cannot be improved for the reasons given above. However CdS linear photosensitive films, with a response-time of less than $10 \mu \mathrm{s}$ are of particular interest compared to CdS films obtained by other processes. ${ }^{6}$

\section{DISCUSSION}

Several authors have studied the response-time of semiconductors. ${ }^{7}$ In a first case, we can introduce a time constant $\tau$ which represents the "free lifetime" corresponding to the time during which the charge carrier is free to contribute to the photoconductivity. The free lifetimes of electrons, $\tau_{n}$, and of holes, $\tau_{p}$, are the main parameters in the photoconductivity processes which are represented by the formula:

$$
\sigma=g e\left(\mu_{n} \tau_{n}+\mu_{p} \tau_{p}\right) \simeq g e \mu_{n} \tau_{n} \text { for } n \text { type }
$$

semi-conductor

where $g$ is the number of electron-holes pairs generated per second in the volume of the photoconductor.

This simplified formula shows how photosensitivity is directly proportional to the free life-time; and in many applications, photosensitivity is frequently increased by incorporating in a material impurity centers which have a smaller probability of capturing majority carriers to bring about recombination. For example in intrinsic cadmium sulphide, called insensitive CdS, the life-time of majority carriers is about $1 \mu \mathrm{s},{ }^{6}$ while in sensitive doped CdS the majority carrier life-time is increased to $1 \mathrm{~ms}$. However, this concept of photosensitivity which is specific to the material, intrinsic or doped, is not sufficient to take into account the mean free path of photoexcited carriers. R. H. Bube introduces the term of photoconductivity gain $G$ which represents the number of charge carriers which pass between the electrodes per second for each photon absorbed per second. ${ }^{6}$

$$
G=\frac{1}{F} \frac{\Delta I}{e}
$$

where $\Delta I=I_{p h}$ (photocurrent) and

$$
F=g . v
$$

where $v=S . L=$ photoconductor volume.

As $I_{p h}=\sigma \frac{S}{L} V_{p h}$, we can write:

$$
G=\frac{1}{g(S . L)}\left(g e \mu_{n} \tau_{n}\right) V_{p h}\left(\frac{S}{L}\right)=\mu_{n} \tau_{n} \frac{V_{p h}}{L^{2}}
$$

and $I_{p h}=F\left(e \mu_{n} \tau_{n}\right) \frac{V_{p h}}{L^{2}}$

This photoconductive gain can also be represented by the ratio of the free life-time $\tau_{n}$ to the transit time $t_{n}$ required for the carrier to move between the electrodes. 


$$
\begin{gathered}
G=\frac{\tau_{n}}{t_{n}} \text { if } t_{n}=\frac{L}{v}=\frac{L}{\mu_{n} E}=\frac{L^{2}}{\mu_{n} V_{p h}} \\
(v=\text { electron velocity })
\end{gathered}
$$

Then, the concept of photoconductive gain can be used to describe why we can obtain a high photoconduction rate. $\left(J_{p h}=\sigma_{p h} E\right)$ and a very fast response time comparatively with other photoconductive CdS films. ${ }^{7}$ The transit time $t_{n}$, proportional to the square of the photoconductor thickness is particularly reduced in very thin films (less than $0.1 \mu \mathrm{m}$ ). In this case, sandwich photocells can be used in modulated light photodetection.

We have to pay particular attention to the application of the photoconductive gain $G$, which, representing the number of charge carriers which pass between the electrodes per second, assumes that the currents passing through the contact obey Ohm's law over a large range of applied voltage.

Several authors ${ }^{8}$ have studied the nature of electrode contacts on cadmium sulphide crystals and, their conclusions show that ohmic contacts could be made with a variety of metals under specific conditions of surface treatments. Sputtering deposition methods, used for $\mathrm{Al} / \mathrm{CdS} / \mathrm{Au}$ sandwich

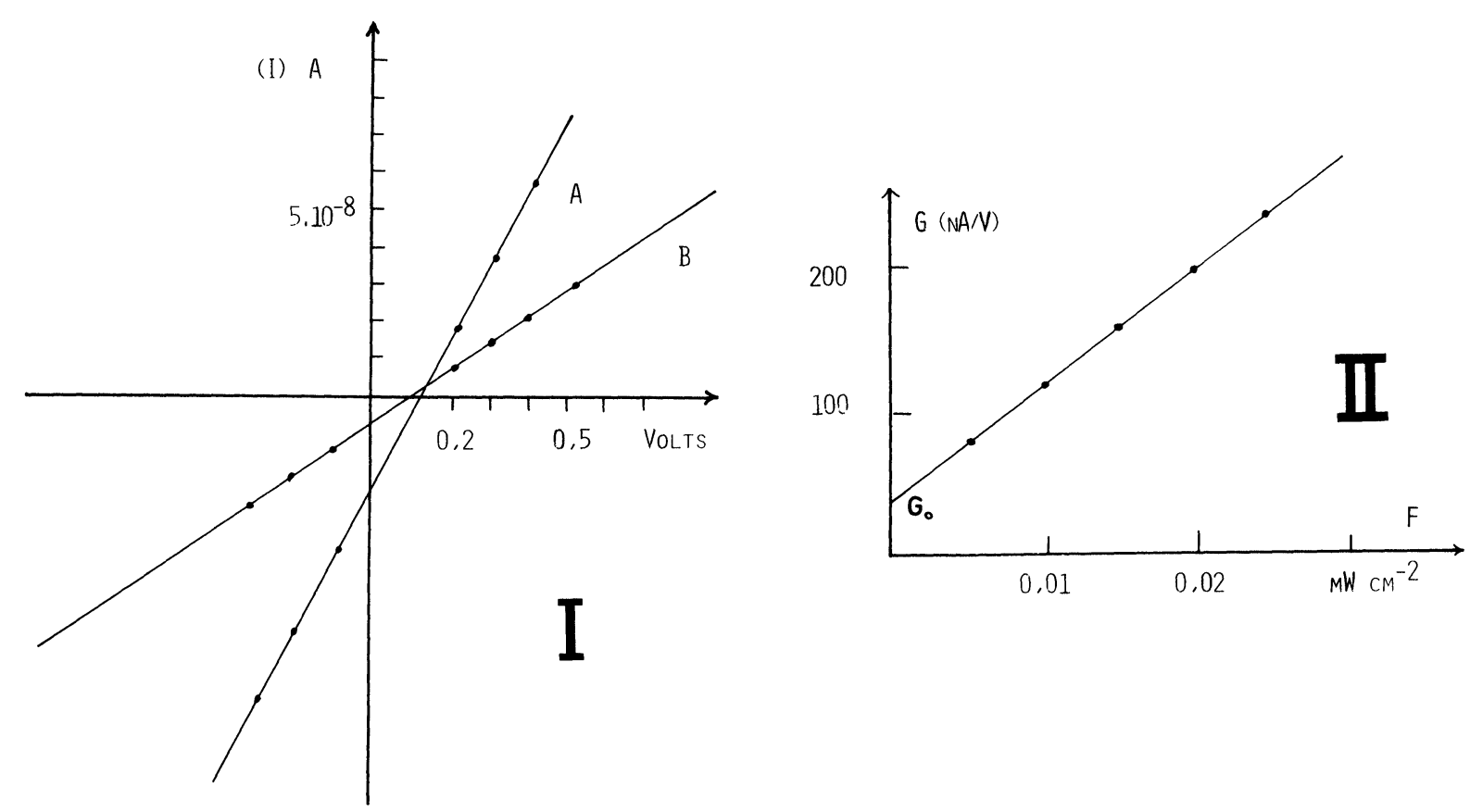

photo cells certainly induce an electronic and ionic bombardment with the glow discharge, which could modify the nature of metal-semi-conductor electrical contacts compared to thermally evaporated films.

In experimental studies made on $\mathrm{Al}-\mathrm{CdS}-\mathrm{Au}$ and $\mathrm{Al}-\mathrm{Zns}-\mathrm{Au}$ sputtered sandwich films ${ }^{9}$ we have shown that the current flow is a bulk limited current explained by the Poole-Frenkel emission, independent of the polarity of the applied voltage. Furthermore, as shown in Figure 4, the observed interband transitions photocurrent perfectly obeys Ohm's law $I=G_{p h}\left(V-V_{p h o}\right)$ where $G_{p h}$ is the photoconductance and $V_{p h o}$, the photo voltage. Experiments also give a linear variation of $G_{p h}$ with the intensity of light; $G_{p h}$ being given by the classical formula of photoconduction:

$$
G_{p h}=\mathrm{e} \mu_{n} \tau_{n} g \frac{S}{L}
$$

These experimental results, show that we have electrode contacts able to supply the free carriers required by the photoconduction process. The photovoltaic effects in sandwich structures can be described by the concept of a photoconductive gain.

FIGURE 4 I: Ohmic variations of photocurrent $I_{p h}=G_{p h}\left(V-V_{p h}\right)$ for different intensities of light A: 0.02 $\mathrm{mW} / \mathrm{cm}^{2}$ B: $0.01 \mathrm{~mW} / \mathrm{cm}^{2}$ II: Linear variations of $G_{p h}$ with the intensity of light. 


\section{CONCLUSION}

This study shows how the response time of sandwich CdS films is particularly dependent on the majority carrier life-time. This is the main difference between sandwich photoconductive cells and PN junctions in which the time constant is essentially due to the minority carrier life-time.

In these structures photo-effects may be represented by a minority carrier injection on both sides of the junction, which involves an important diffusion current density.

This property arises also from the general description of photovoltaic effects in sandwich cells.

In $\mathrm{PN}$ junctions the total current can be written:

$$
\begin{aligned}
& I_{T}=I_{0}\left[\exp \left\{+\frac{e V}{k T}\right\}-1\right]+I_{p h}(F) \\
& I_{T}=f(V)+I_{p h}(F)
\end{aligned}
$$

and so the photoinjection appears only in the complementary $I_{p h}(F)$ which does not affect the voltage dependent term $f(V)$; whereas in sandwich cells, the total current can be written. ${ }^{3}$

$$
\begin{aligned}
& I_{T}=\left(G_{p h}+G_{0}\right) V+\left(G_{p h}+G_{0}\right) V_{i} \\
& I_{T}=\left(G_{p h}+G_{0}\right) V+I_{p h} \\
& I_{T}=f(F, V)+I_{p h}(F) .
\end{aligned}
$$

In this case the photoinjection appears in the complementary term $I_{p h}$ as well as in the voltagedependent term $f(F, V)$. We can say that this photovoltaic effect results from the conductance variations in a built-in electric field.

This explains the importance of majority carrier life-time in response time, as can be observed in photoresistance properties. ${ }^{4,6}$ In this condition it is worthwhile to use (as photodetectors) very thin films for which the transit time $t_{n}$ is considerably reduced.

\section{APPENDIX}

Decay of electron and hole current densities.

\section{BASIC EQUATIONS}

$$
\begin{aligned}
& \frac{\partial n(x, t)}{\partial t}=\frac{1}{e} \frac{\partial J_{n}}{\partial x}(x, t)+g(t)-\frac{n(x, t)}{\tau_{n}} \\
& \frac{\partial p(x, t)}{\partial t}=-\frac{1}{e} \frac{\partial J_{p}}{\partial x}(x, t)+g(t)-\frac{p(x, t)}{\tau_{p}}
\end{aligned}
$$

(3) $J_{n}(x, t)=e \mu_{n} E n(x, t)+e D_{n} \frac{\partial n(x, t)}{\partial x}$

(4) $J_{p}(x, t)=e \mu_{p} E p(x, t)-e D_{p} \frac{\partial p(x, t)}{\partial x}$

$g(t)=$ number of electrons holes pairs created by illumination $(g(t)$ can be supposed independent of $x$ for photoconductor thickness less than $0.1 \mu \mathrm{m}$ )

\section{NEW EQUATIONS GIVEN BY LAPLACE TRANSFORM}

$$
\begin{aligned}
& \widetilde{F}(x, \alpha)=\int_{0}^{\infty} \exp ^{-\alpha t} f(x, t) d t \quad F(x, \alpha)=L f(x, t) \\
& \tilde{N}(x, \alpha)=\int_{0}^{\infty} \exp ^{-\alpha t} n(x, t) d t \\
& \widetilde{P}(x, \alpha)=\int_{0}^{\infty} \exp ^{-\alpha t} p(x, t) d t \\
& \widetilde{I}_{n}(x, \alpha)=\int_{0}^{\infty} \exp ^{-\alpha t} J_{n}(x, t) d t \\
& \widetilde{I}_{p}(x, \alpha)=\int_{0}^{\infty} \exp ^{-\alpha t} J p(x, t) d t \\
& \tilde{Y}(\alpha)=\int_{0}^{\infty} \exp ^{-\alpha t} g(t) d t \\
& \text { (5) }\left\{\alpha+\frac{1}{\tau_{n}}\right\} \tilde{N}(x, \alpha)=\frac{1}{e} \frac{\partial \tilde{I}}{\partial x} n(x, \alpha)+\tilde{Y}(\alpha)+n(x, 0) \\
& \text { (6) }\left\{\alpha+\frac{1}{\tau_{p}}\right\} \tilde{P}(x, \alpha)=-\frac{1}{e} \frac{\partial \tilde{l}}{\partial x} p(x, \alpha) \\
& \quad+\tilde{Y}(\alpha)+p(x, 0)
\end{aligned}
$$

Eq. (3) becomes

$$
\begin{gathered}
D_{n} \frac{\partial^{2} \widetilde{I}_{n}(x, \alpha)}{\partial x^{2}}+\mu_{n} E \frac{\partial \tilde{I}_{n}(x, \alpha)}{\partial x}-\left(\alpha+\frac{1}{\tau_{n}}\right) \tilde{I}_{n}(x, \alpha)= \\
-e \mu_{n} E \tilde{Y}(\alpha)-e \mu_{n} E n(x, 0)-e D_{n} \frac{\partial n(x, 0)}{\partial x}
\end{gathered}
$$

Eq. (4) becomes

$$
\begin{aligned}
& D_{p} \frac{\partial^{2} I_{p}(x, \alpha)}{\partial x^{2}}-\mu_{p} E \frac{\partial \tilde{I}_{p}(x, \alpha)}{\partial x}-\left(\alpha+\frac{1}{\tau_{p}}\right) \tilde{I}_{p}(x, \alpha)= \\
& -e \mu_{p} E \tilde{Y}(\alpha)-e \mu_{p} E p(x, 0)+e D_{p} \frac{\partial p(x, 0)}{\partial x}
\end{aligned}
$$


Equations (7) and (8) are second order linear differential equations. Their solutions may be written by $\widetilde{I}_{n}=\widetilde{I}_{n o}+\widetilde{I}_{n G} \widetilde{I}_{p}=\widetilde{I}_{p o}+\widetilde{I}_{p G}$

where

$\tilde{I}_{n o}$ and $\tilde{I}_{p o}$ are solutions of Eq's (7) and (8) when $\widetilde{Y}=0$.

$\tilde{I}_{n G}$ and $\tilde{I}_{p G}$ are particular solutions for $\tilde{Y} \neq 0$

Thus, $\widetilde{I}_{n o}+\widetilde{I}_{p o}$ represents the Laplace transform $\mathscr{L} J_{d c}$ of the dark current and $\widetilde{I}_{n G}+\widetilde{I}_{p G}$, the Laplace transform $\mathscr{L} J_{p h}$ of the photocurrent given by $J_{p h}{ }^{(t)}=\mathscr{L}^{-1}\left[I_{n G}+\widetilde{I}_{p G}\right]$.

\section{PHOTOCURRENT CALCULATION}

a) $g(t)$ is a $\delta(t)$ function.

$$
\begin{aligned}
& g(t)=\frac{1}{\tau_{0}} \text { for } 0<t<\tau_{0} \\
& g(t)=0 \text { for } \tau_{0}<t \text { then } \tilde{Y}(\alpha)=g_{0}
\end{aligned}
$$

Then the particular solutions $\tilde{I}_{n g}$ and $\tilde{I}_{p g}$ are:

$$
\begin{aligned}
\tilde{I}_{n g}= & \frac{e \mu_{n} E g_{0}}{\alpha+\frac{1}{\tau_{n}}} \tilde{I}_{p g}=\frac{e \mu_{p} E g_{0}}{\alpha+\frac{1}{\tau_{p}}} \\
& J_{p h}(t)=e E g_{0}\left(\mu_{n} \exp ^{-t / \tau} n+\mu_{p} \exp ^{-t / \tau} p\right)
\end{aligned}
$$

b) $g(t)$ is an exponential function

$$
g(t)=g_{0} \exp ^{-t / \tau} 0 \text { then } \tilde{Y}(\alpha)=\frac{g_{0}}{\alpha+\frac{1}{\tau_{0}}}
$$

$$
\tilde{I}_{n g}=\frac{e \mu_{n} E g_{0}}{\left(\alpha+\frac{1}{\tau_{n}}\right)\left(\alpha+\frac{1}{\tau_{0}}\right)} I_{p g}=\frac{e \mu_{p} E g_{0}}{\left(\alpha+\frac{1}{\tau_{p}}\right)\left(\alpha+\frac{1}{\tau_{0}}\right)}
$$

$$
\begin{aligned}
J_{p h}(t) & =e E g_{0}\left[\mu_{n} \frac{\tau_{n} \exp ^{-t / \tau} n-\tau_{0} \exp ^{-t / \tau} 0}{\tau_{n}-\tau_{0}}\right. \\
& \left.+\mu_{p} \frac{\tau_{p} \exp ^{-t / \tau} p-\tau_{0} \exp ^{-t / \tau} 0}{\tau_{p}-\tau_{0}}\right]
\end{aligned}
$$

\section{REFERENCES}

1. Semi-conductor photoelectric devices. (London Illife Books Ltd) 1968. p. 244.

2. H. Murray, A. Tosser, 6th Int. Symp. on photon detectors Siofok Hungary. September 16-19 1974.

3. A. Piel, H. Murray, Revue de Physique Appliquée, Tome 11, Mai 1976, p. 397-402.

4. R. H. Bube, Photoconductivity of solids, p. 326 (John Wiley and Sons, Princeton, New Jersey), 1960.

5. S. M. Rykvin, Photoelectric effects in semi-conductors (Consultants Bureau New York) 1964, p. 63-72.

6. R. H. Bube, Photoconductivity of solids, p. 59-60 (John Wiley and Sons, Princeton, New Jersey), 1960.

7. See in Photoelectronic materials and devices (Van Nostrand Co, Princeton, New Jersey) 1965. Chapter 2.

8. R. H. Bube Photoconductivity of solids (John Wiley and Sons, Princeton, New Jersey) Chapter 5.

9. A. Tosser, H. Murray, M. Arsalane, A. Piel 7th Symp. on photon detectors Brauschweig F.R.G. 17-21 May 1976. 

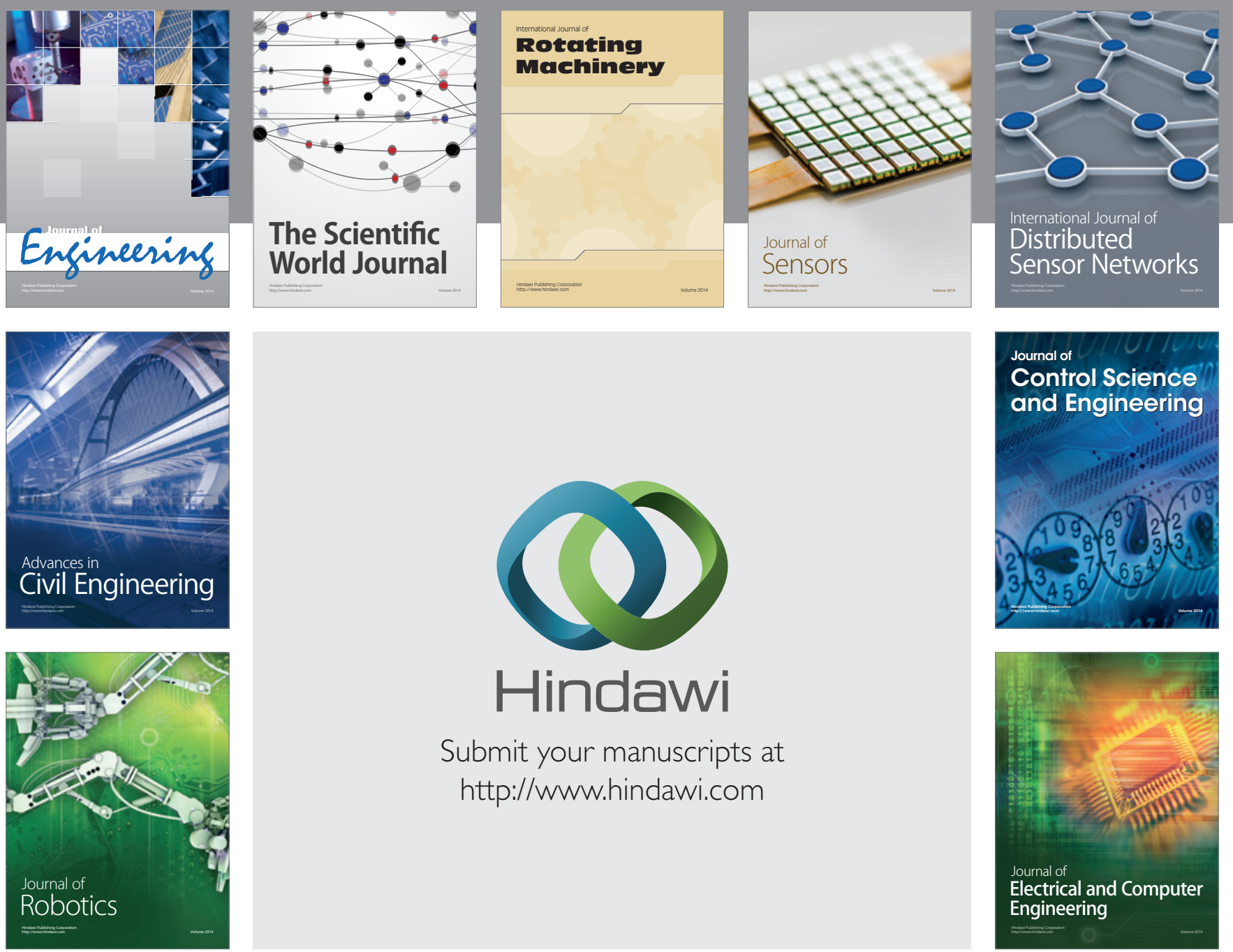

Submit your manuscripts at

http://www.hindawi.com
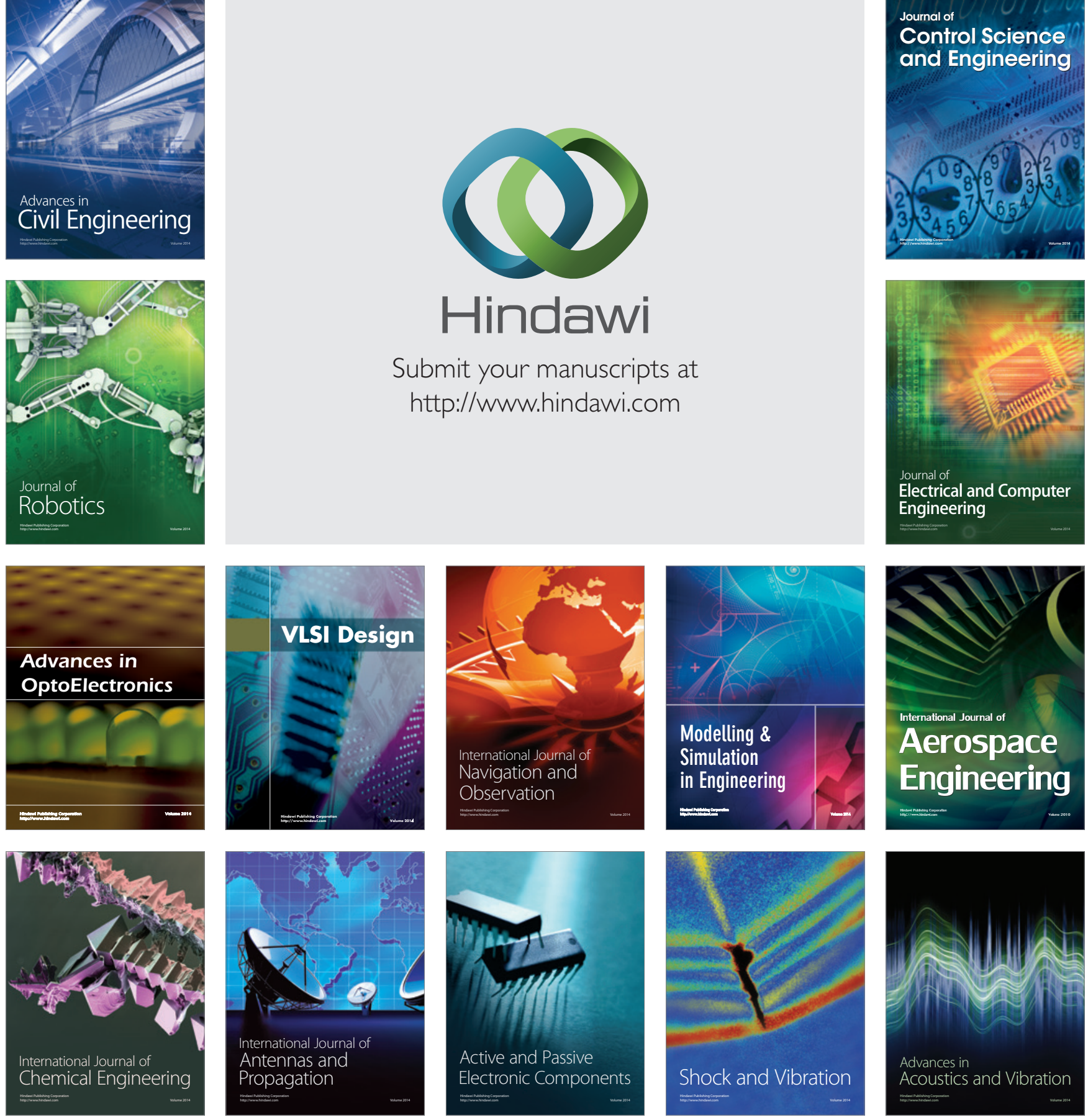